\title{
Efeitos da estimulação diafragmática elétrica transcutânea na função pulmonar em idosos
}

\author{
Diaphragmatic effects of the transcutaneous electrical stimulation on the pulmonary function \\ in elderly
}

\section{Resumo}

Introdução: O envelhecimento promove mudanças em diversos sistemas orgânicos, inclusive no respiratório, evidenciando alterações nos centros reguladores da ventilação, nos quimiorreceptores, na densidade óssea da caixa torácica, além de diminuição da força dos músculos inspiratórios. Objetivo: Avaliar o efeito de um programa de treinamento específico da musculatura respiratória por meio da estimulação diafragmática elétrica transcutânea (EDET) sobre a função pulmonar de idosos. Método: 21 idosos foram divididos em dois grupos: controle (GC), composto

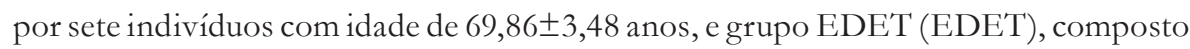
por 14 voluntários com idade de 71,36 $\pm 4,73$ anos, submetidos ao treinamento com EDET durante duas semanas. Avaliaram-se os volumes e capacidades pulmonares, como volume corrente (VC), volume minuto (VM), capacidade inspiratória (CI) e capacidade vital (CV) por meio da ventilometria e a força da musculatura respiratória antes da primeira e após a décima sessão, por meio da avaliação das pressões respiratórias máximas. Utilizaram-se os testes de Wilcoxon e Mann-Whitney para as devidas comparações estatísticas, sendo adotado um nível de significância de 5\%. Resultados: O grupo EDET apresentou incremento na pressão inspiratória máxima (PImáx), na pressão expiratória máxima (PEmáx) e no volume corrente (VC) após o treinamento. Conclusão: O treinamento muscular mediante utilização da EDET promoveu melhora da força muscular respiratória e do volume corrente. Registro de ensaio clínico: RBR-5dmpkr.

\section{Abstract}

Introduction: Aging causes changes in several organ systems, including the respiratory system, highlighting changes in the respiratory control centers, chemoreceptors, bone density on the rib cage and decreased elasticity of the respiratory muscles. Objective: To evaluate the effect of a program of respiratory muscle training through transcutaneous

\footnotetext{
Programa de Pós-graduação em Fisioterapia, Faculdade de Ciências da Saúde. Universidade Metodista de Piracicaba. Piracicaba, SP, Brasil.

2 Curso de Fisioterapia, Faculdade de Ciências da Saúde. Universidade Metodista de Piracicaba. Piracicaba, SP, Brasil.
}

Laisa Antonela dos Santos Juliana Rabechi Borgi² Jorge Luiz Nogueira Daister Eli Maria Pazzianotto-Forti'

Palavras-chave: Diafragma. Estimulação Elétrica. Envelhecimento. 
electrical diaphragmatic stimulation (TEDS) on pulmonary function in the elderly. Method: Twenty-one seniors were divided into two groups: control (CG), composed of seven individuals with mean age of $69.86 \pm 3.48$ years, and experimental group (EG), composed of 14 volunteers with a mean age of $71.36 \pm 4.73$ years, submitted to TEDS training for two weeks. We evaluated the volumes and lung capacities, as tidal volume (VT), minute volume (MV), inspiratory capacity (IC) and vital capacity (VC) and respiratory muscle strength in the first and tenth session. The Wilcoxon and Mann-Whitney test was used for appropriate statistic comparisons, adopting a significance level of 5\%. Results: In intragroup comparisons, only EG showed an increase in maximal inspiratory pressure (MIP), maximal expiratory pressure (MEP) and tidal volume (VT) after training. Conclusion: Muscle training through use of TEDS promoted improvement in respiratory muscle strength and tidal volume. Trial registration: RBR-5dmpkr.

\section{INTRODUÇÃO}

O envelhecimento pode ser definido como um processo dinâmico e progressivo no qual há alterações morfológicas, fisiológicas e biológicas que vão degradando o organismo, afetando também o sistema respiratório e as estruturas que o envolvem. As alterações incluem os centros respiratórios, quimiorreceptores, configurações torácicas e diminuição da força dos músculos respiratórios. ${ }^{1,2}$

O envelhecimento produz diminuição do número de unidades motoras e de neurônios e do volume das fibras musculares esqueléticas de tipos I e II, resultando numa redução da força muscular. No entanto, tais efeitos deletérios podem ser atenuados com o treinamento específico da musculatura respiratória. ${ }^{3}$ A estimulação diafragmática elétrica transcutânea (EDET) é um método disponível na fisioterapia respiratória cujo objetivo é melhorar a condição muscular, favorecendo a resistência e a força dos músculos respiratórios. ${ }^{4,5}$

Estudo $^{6}$ que avaliou os efeitos morfológicos e histológicos da ação da EDET em ratos constatou que a corrente elétrica utilizada no método não promove alterações no traçado eletrocardiográfico, além de ter sido observado aumento no nível de glicogênio no grupo que foi estimulado com a corrente. $\mathrm{O}$ aumento do glicogênio implica melhora das reservas
Key words: Diaphragm. Electric Stimulation. Aging. energéticas e eficácia quimiometabólica, proporcionando ao músculo maior fonte de energia para realizar as contrações.

A EDET, além de interferir no ganho de força e resistência muscular respiratória, garante a prevenção de hipotrofia muscular. ${ }^{7}$ Sua aplicação proporciona melhora da excursão do músculo diafragma, bem como aumento nos valores espirométricos. ${ }^{8}$ Segundo o exposto, aventa-se a hipótese de que a EDET possa trazer benefícios para a musculatura respiratória dos idosos e aumento dos volumes e capacidades pulmonares.

Justifica-se este estudo em função da escassez de trabalhos encontrados na literatura sobre o treinamento muscular respiratório com EDET em idosos. O objetivo foi avaliar o efeito de um programa de treinamento respiratório pautado na estimulação diafragmática elétrica transcutânea sobre os volumes e capacidades pulmonares e força muscular respiratória de indivíduos idosos saudáveis.

\section{MATERIAL E MÉTODOS}

Os voluntários foram triados numa instituição de longa permanência para idosos de Piracicaba, Estado de São Paulo, por meio de convite para participar da pesquisa. Os dados foram coletados entre março e junho de 2010. 
A amostra foi composta por 21 voluntários saudáveis, do gênero masculino, com faixa etária compreendida entre 67 e 79 anos $(70,86 \pm 4,33)$, não fumantes, não praticantes de exercícios físicos regulares e sem história de doença pulmonar, doenças neuromusculares ou alterações posturais que pudessem comprometer a musculatura respiratória ou doenças cardiovasculares (com exceção da hipertensão controlada por medicamento).

Os voluntários foram divididos em grupo controle (GC), com sete sujeitos que receberam EDET no limiar sensitivo, sem proporcionar contração muscular; e grupo EDET, composto por 14 idosos tratados com EDET, a qual promoveu contração muscular palpável.

Todos os voluntários incluídos no estudo foram submetidos a duas avaliações, sendo a primeira antes do tratamento proposto, no qual se coletaram medidas antropométricas, sinais vitais, história clínica, hábitos de vida (tabagismo e atividade física regular), avaliação dos volumes e capacidades pulmonares, por meio da ventilometria, e da força muscular respiratória, pelas medidas das pressões respiratórias máximas. $\mathrm{Na}$ segunda avaliação, após o término do tratamento, as medidas dos volumes e capacidades pulmonares e da força muscular respiratória foram repetidas.

As medidas de pressão inspiratória máxima (PImáx) e pressão expiratória máxima (PEmáx) foram realizadas por meio de um manovacuômetro analógico (GER-AR, SP, Brasil), com intervalo operacional de 0 a $\pm 300 \mathrm{cmH}_{2} \mathrm{O}$.

Para a realização das medidas da PEmáx, os voluntários foram orientados a realizar uma inspiração profunda, ao nível da capacidade pulmonar total (CPT) e, em seguida, uma expiração máxima no bocal. A PImáx foi medida após uma expiração máxima até próximo ao volume residual (VR) e, em seguida, realizada uma inspiração máxima no bocal. Essas manobras foram sustentadas por pelo menos dois segundos. Após um período de treinamento, suficiente para o aprendizado da técnica, cada voluntário executou três medidas de inspiração máximas consideradas tecnicamente satisfatórias, isto é, com duração mínima de 2 s, sem escape de ar e com valores próximos entre si $(<10 \%)$, computando-se o maior valor absoluto obtido. ${ }^{?}$

Para a realização das medidas de volume corrente (VC), volume minuto (VM), capacidade inspiratória (CI) e capacidade vital (CV), foi utilizado o equipamento ventilômetro (OHMEDA Respirometer ${ }^{\circledR}$ RM121, Tóquio, Japão). Os voluntários permaneceram sentados, segurando a máscara de silicone envolvendo sua cavidade nasal e oral, evitando o escape de ar. Para a avaliação do VC, VM e CV, foi utilizado o ramo expiratório do ventilômetro; para a aferição da CI, utilizou-se o ramo inspiratório do equipamento. ${ }^{10}$

Para a avaliação do VC, o voluntário foi orientado a realizar o ciclo respiratório normal. O equipamento foi disparado no momento da inspiração, para que o volume expirado fosse registrado. Para a avaliação do VM, o voluntário recebeu a mesma orientação, porém permaneceu respirando por 60 segundos, sendo que ao mesmo tempo, com o auxílio de um cronômetro, foi aferida a frequência respiratória. Para as medidas da CV, os voluntários foram orientados a realizar uma inspiração máxima, seguida de uma expiração máxima e lenta, no ramo expiratório do ventilômetro.

Para as medidas da CI, o voluntário foi orientado a realizar uma inspiração máxima a partir de uma expiração normal, sendo essa medida registrada no ramo inspiratório do ventilômetro. $^{10}$

Para a realização da EDET, foi utilizado o equipamento Dualpex 994, modelo Phrenics $\left(\right.$ Quark $^{\circledR}$, Piracicaba, SP, Brasil), dotado de microprocessador, versão $1.6 \mathrm{~B}$.

O treinamento muscular respiratório foi realizado uma vez ao dia, por 30 minutos, cinco dias por semana, durante duas semanas. ${ }^{11}$ Para a aplicação da técnica, os voluntários ficaram em decúbito dorsal, com os joelhos semiflexionados, pés apoiados, braços ao longo do corpo e a cabeça 
sobre um travesseiro. Os eletrodos foram fixados à pele, previamente limpa com solução alcoólica a $70 \%$, por meio de fita micropore. Entre a pele e o eletrodo foi aplicado gel condutor.

Foram utilizados quatro eletrodos de silicone carbono $(4 \mathrm{x} 4 \mathrm{~cm})$, sendo posicionados dois na região paraesternal, ao lado do processo xifóide, no sentido das fibras musculares; os outros dois eletrodos foram fixados nos pontos motores do músculo diafragma, entre o $6^{\circ}$ e $7^{\circ}$ espaços intercostais, nas linhas axilares anterior direita e esquerda, paralelos às fibras musculares. ${ }^{12}$

A determinação dos pontos de colocação dos eletrodos foi obtida por meio da palpação, com o voluntário em decúbito dorsal. Após a demarcação do local, o ponto de colocação do eletrodo foi confirmado pela contração muscular, no início do desencadear da corrente elétrica e no grupo controle, pelo feedback do voluntário.

Os parâmetros adotados para realização da EDET foram: corrente despolarizada com frequência de pulso de $30 \mathrm{~Hz}$, frequência respiratória de $14 \mathrm{rpm}$, tempo de subida (rampa) de $0,7 \mathrm{~s}$, largura de pulso de $1,2 \mathrm{~ms} .^{12}$ Foram realizadas dez sessões consecutivas, com tempo de duração de 30 minutos cada. A intensidade foi determinada de acordo com os grupos, respeitando-se o limiar sensitivo para o GC e o limiar motor para o grupo EDET. ${ }^{11}$

A análise estatística foi realizada por meio do teste de normalidade Shapiro Wilk, utilizando uma metodologia não paramétrica por meio dos testes Wilcoxon e Mann-Whitney. Considerouse um $\mathrm{p}<0,05$ para todas as comparações. $\mathrm{O}$ processamento dos dados foi realizado por meio do software BioEstat, versão 5.0.

Os idosos foram esclarecidos quanto aos objetivos do estudo e assinaram um Termo de Consentimento Livre e Esclarecido, sendo os procedimentos do estudo aprovados pelo Comitê de Ética em Pesquisa da Universidade Metodista de Piracicaba, sob o protocolo $\mathrm{n}^{\circ}$ 08/05, obedecendo aos requisitos da Declaração de Helsinque (Emenda de 2000) e a Resolução 196/96 do Conselho Nacional de Saúde.

\section{RESULTADOS}

A idade e as características antropométricas dos voluntários que constituíram os grupos estudados estão apresentadas na tabela 1.

Tabela 1 - Médias e desvio-padrão da idade e das características antropométricas dos voluntários estudados no grupo controle (GC) e no grupo EDET (EDET). Piracicaba, SP, 2010.

\begin{tabular}{lcc}
\hline \multicolumn{1}{c}{ Variáveis } & GC & EDET \\
\hline Idade (anos) & $69,86 \pm 3,48$ & $71,36 \pm 4,73$ \\
Massa corporal $(\mathrm{kg})$ & $81,00 \pm 9,27$ & $80,96 \pm 13,58$ \\
Altura (m) & $1,72 \pm 0,03$ & $1,69 \pm 0,07$ \\
Índice de massa corporal $\left(\mathrm{kg} / \mathrm{m}^{2}\right)$ & $27,36 \pm 3,28$ & $28,46 \pm 4,35$ \\
\hline
\end{tabular}

Em relação à PImáx e PEmáx, na comparação do momento anterior e posterior à intervenção fisioterapêutica em cada grupo, constatou-se aumento significativo para essas variáveis no grupo EDET (tabela 2). Quando comparados o GC e o EDET, antes das intervenções estes não apresentaram diferença significativa. Houve, no entanto, aumento significativo nas pressões respiratórias no EDET, quando comparado ao GC. Os valores da avaliação da força muscular respiratória estão expressos na tabela 2. 
Tabela 2 - Médias e desvio-padrão das medidas de pressão inspiratória máxima (PImáx) e pressão expiratória máxima (PEmáx) do grupo controle (GC) e do grupo EDET (EDET). Piracicaba, SP, 2010.

\begin{tabular}{|c|c|c|c|}
\hline Variáveis & & $1^{\text {a }}$ avaliação / Pré-TTO & $2^{\text {a }}$ avaliação / Após TTO \\
\hline \multirow[t]{2}{*}{ PImáx $\left(\mathrm{cmH}_{2} \mathrm{O}\right)$} & GC & $-34,29 \pm 10,18$ & $-32,91 \pm 17,39$ \\
\hline & EDET & $-42,14 \pm 12,67$ & $-55,71 \pm 12,84^{*} \#$ \\
\hline \multirow[t]{2}{*}{ PEmáx $\left(\mathrm{cmH}_{2} \mathrm{O}\right)$} & GC & $60,71 \pm 18,80$ & $59,95 \pm 21,85$ \\
\hline & EDET & $63,21 \pm 19,18$ & $83,57 \pm 20,52^{* \#}$ \\
\hline
\end{tabular}

*Comparação intragrupo $(\mathrm{p}<0,05)$; \# comparação entre o GC na segunda avaliação e o grupo EDET após tratamento $(\mathrm{p}<0,05)$; TTO: tratamento.

Os resultados da ventilometria evidenciaram aumento significativo no grupo EDET apenas na variável VC. Quando comparados os dois grupos, houve diferença significativa do VC na comparação entre GC e GE antes da intervenção, sem diferenças nas outras variáveis, assim como mostram os resultados apresentados na tabela 3.

Tabela 3 - Média e desvio-padrão das medidas de volume corrente (VC), volume minuto (VM), capacidade vital (CV) e capacidade inspiratória (CI) do grupo controle (GC) e do grupo EDET (EDET). Piracicaba, SP, 2010.

\begin{tabular}{cccc}
\hline Variáveis & & $1^{a}$ avaliação / Pré-TTO & $2^{a}$ avaliação / Após TTO \\
\hline VC $(\mathrm{mL})$ & GC & $348,57 \pm 81,53$ & $340,01 \pm 110,14$ \\
& EDET & $411,43 \pm 79,79$ & $453,24 \pm 164,93^{*} \#$ \\
VM $(\mathrm{L})$ & GC & $6,64 \pm 1,99$ & $6,31 \pm 2,50$ \\
& EDET & $7,48 \pm 1,53$ & $8,47 \pm 1,96$ \\
CV $(\mathrm{L})$ & GC & $1,79 \pm 0,60$ & $1,77 \pm 0,71$ \\
& EDET & $2,40 \pm 0,62$ & $2,56 \pm 0,44$ \\
CI (L) & GC & $1,25 \pm 0,37$ & $1,25 \pm 0,49$ \\
& EDET & $1,82 \pm 0,71$ & $2,10 \pm 0,49$ \\
\hline
\end{tabular}

*Diferença significativa após o tratamento $(\mathrm{p}<0,05)$; \#diferença significativa entre o GC na segunda avaliação e o grupo EDET após tratamento ( $\mathrm{p}<0,05)$; TTO: tratamento.

\section{DISCUSSÃO}

O propósito deste estudo foi testar a hipótese de que o treinamento muscular respiratório com a EDET em idosos sedentários traria benefícios a essa musculatura. Os resultados obtidos evidenciaram melhora da força muscular inspiratória, expiratória e do volume corrente nos idosos.
A idade predispõe todos os sistemas do organismo a um declínio progressivo da capacidade funcional, e é comum a diminuição da força muscular. ${ }^{13}$ Pesquisas anteriores apontam alterações na normalidade dos tecidos biológicos e, consequentemente, declínio dos sistemas fisiológicos do organismo, em decorrência do envelhecimento. ${ }^{1,14,15}$ 
No sistema respiratório, ocorre redução lenta e progressiva da complacência da parede torácica, do aumento da resistência das vias aéreas, ${ }^{16}$ tendo início a sarcopenia, definida como o declínio progressivo de massa muscular, força e qualidade de contração. ${ }^{15}$ Oyarzun ${ }^{16}$ destaca que a sarcopenia ocorre em função da hipoplasia das fibras musculares, alterações nos neurônios periféricos, além de modificações intrínsecas à célula: no transporte de cálcio nas fibras musculares, redução do ATP mitocondrial e redução na síntese de miosina de cadeia pesada.

Além das alterações provocadas pela sarcopenia, existem comorbidades que podem decorrer de tal alteração, tais como riscos de distúrbios metabólicos (diabetes tipo II) e osteoporose. Assim, o treinamento muscular respiratório é considerado uma importante ação para reverter o decréscimo de força muscular. ${ }^{14}$

Watsford et al. ${ }^{17}$ tiveram por objetivo verificar o papel do exercício habitual na função dos músculos respiratórios em idosos ativos e inativos. Os resultados demonstraram que os indivíduos ativos obtiveram maior distância percorrida no teste de caminhada e manutenção da capacidade respiratória, o que permite maior qualidade de vida. Os autores destacaram a importância de estudar treinamentos específicos para a musculatura respiratória em idosos, além de ressaltarem o risco aumentado de comorbidades para idosos inativos.

Com o envelhecimento, pode ocorrer redução do pico de fluxo expiratório (PFE), comprometendo a tosse. Esta é considerada um mecanismo complexo que protege a via aérea de partículas estranhas e secreções, reduzindo o risco de infecções no trato respiratório; ${ }^{18}$ sendo de muita importância para os idosos. Freitas et al. ${ }^{19}$ inferiram que idosos mais ativos apresentam melhores condições de força muscular respiratória e de PFE - consequentemente, menor risco de infecções.

Assim, o treinamento específico da musculatura se torna indispensável para garantir a qualidade de vida. Pesquisadores buscam técnicas, procedimentos e tratamentos que possam promover melhoria na qualidade de vida dessa condição inerente ao ser humano, que é o envelhecimento. ${ }^{20}$ Envelhecer com qualidade de vida significa realizar as atividades diárias de maneira normal e possuir independência funcional. ${ }^{21}$

Um protocolo fisioterapêutico associado à EDET foi aplicado a pacientes em pós-operatório (PO) de revascularização do miocárdio e observou-se que no quinto dia de $\mathrm{PO}$ os valores obtidos eram superiores aos do segundo dia de PO, embora menores do que no pré-operatório. Os autores observaram que a intervenção proposta promoveu uma recuperação mais precoce dos pacientes após a cirurgia. ${ }^{22}$

Ao analisar o efeito da EDET em relação à função diafragmática, foram estudadas 20 mulheres jovens saudáveis, e assim como no presente estudo, avaliaram-se a força muscular e os volumes e capacidades pulmonares. Os resultados obtidos evidenciaram aumento nos valores de PImáx, VC, VM e CI, os quais se mostraram coerentes com os valores encontrados neste estudo. O treinamento com a EDET pode ser uma ferramenta efetiva na fisioterapia respiratória, promovendo melhora no desempenho do músculo diafragma. ${ }^{23}$

Estudo com idosas sedentárias e ativas verificou a influência da atividade física no incremento da força muscular inspiratória e expiratória. Ao comparar os resultados da pesquisa com os valores preditos pela literatura, foi observado incremento na força muscular respiratória nas praticantes de atividade física, ${ }^{24}$ o que consequentemente preveniu o declínio funcional dessa musculatura respiratória. Os autores destacam a importância de um treino específico para melhorar o desempenho dessa musculatura específica. ${ }^{25}$

Em relação ao volume corrente, houve incremento desta variável nos idosos que realizaram treinamento com a EDET. Este achado pode estar relacionado ao fato de que, com o ganho de força muscular, a musculatura 
respiratória passou a realizar contrações mais efetivas, facilitando a ventilação pulmonar e ampliando o volume corrente. ${ }^{26}$

O treinamento da musculatura respiratória tem se mostrado benéfico quanto à melhora das capacidades pulmonares. Tal como encontrado no presente estudo, pesquisa realizada com idosas entre 60 e 69 anos, após o programa de treinamento muscular respiratório, avaliou por meio da espirometria os volumes e capacidades pulmonares, observando melhorias de até $30 \%$ no grupo treinado, além de progresso no desempenho físico, indicando melhor qualidade de vida. ${ }^{22}$

Os resultados obtidos neste estudo evidenciaram melhora da força muscular inspiratória e expiratória em idosos por meio do treinamento realizado com a EDET. Tais achados corroboram a literatura, segundo a qual trabalhos apresentam os benefícios adquiridos pela EDET, como melhora nas condições energéticas e aumento da força da musculatura respiratória., ${ }^{411,12}$

\section{REFERÊNCIAS}

1. Janssens JP, Pache JC, Nicod LP. Physiological changes in respiratory function associated with ageing. Eur Respir J 1999;13(1):197-205.

2. Waters DL, Baumgartner RN, Garry PJ, Vellas B. Advantages of dietary, exercise-related, and therapeutic interventions to prevent and treat sarcopenia in adult patients: an update. Clin Interv Aging 2010;5:259-70.

3. Lazzoli JK, Oliveira MAB, Leitão MB, Nóbrega ACL, Nahas RM, Rezende L, et al. Posicionamento oficial da Sociedade Brasileira de Medicina do Esporte sobre: esporte competitivo em indivíduos acima de 35 anos. Rev Bras Med Esporte 2001;7(3):83-92.

4. Nascimento P, Aquim EE. A aplicação da eletroestimulação transcutânea diafragmática em indivíduos normais. Fisioter Bras 2000;1(1):15-8.

5. Geddes LA, Voorhees WD, Bourland JD, Riscili CE. Optimum stimulus frequency for contracting the inspiratory muscles with chest-surface electrodes to produce artificial respiration. Ann Biomed Eng 1990;18(1):103-8.
O número de voluntários que participaram da pesquisa foi uma limitação do estudo, pois a amostra utilizada foi referente apenas aos idosos residentes em uma única instituição de longa permanência, sendo muitos excluídos no processo de triagem para a inclusão no estudo.

\section{CONCLUSÃO}

Conclui-se que o treinamento muscular respiratório realizado com a estimulação diafragmática elétrica transcutânea (EDET) promoveu melhora da força muscular inspiratória e expiratória, com consequente incremento no volume corrente, podendo ser considerado um recurso efetivo da fisioterapia respiratória para ser utilizado com indivíduos idosos.

Outros estudos utilizando a EDET devem ser realizados, uma vez que o recurso pode potencializar a musculatura respiratória de idosos e assim ser incluído como estratégia preventiva contra o declínio da força muscular respiratória e da função pulmonar.

6. Cancelliero KM, Costa D, Silva CA. Estimulação diafragmática elétrica transcutânea melhora as condições metabólicas dos músculos respiratórios de ratos. Rev Bras Fisioter 2006;10(1):59-65.

7. Ayas NT, McCool FD, Gore R, Lierberman SL, Brown R. Prevention of human diaphragm atrophy with short periods of electrical stimulation. Am J Respir Crit Care Med 1999;159(6):2018-20.

8. Cuello AF, Masciantonio L, Mendoza SM. Estimulación diafragmática eléctrica transcutánea. Med Intensiva 1991;8(4):194-202.

9. Simões RP, Castello V, Auad MA, Dionísio J, Mazzonetto M. Prevalence of reduced respiratory muscle strength in institutionalized elderly people. São Paulo Med J 2009;127(2):78-83.

10. Paisani DM, Chiavegato LD, Faresin SM. Volumes, capacidades pulmonares e força muscular respiratória no pós-operatório de gastroplastia. J Bras Pneumol 2005;31(2):125-32. 
11. Criner GJ, Travaline JM, Holt GA, Bosse CG, Kelsen SG. Variability of electrophrenic diaphragm twitch stimulation over time in normal subjects. Respir Physiol 1999;118(1):39-47.

12. Costa D, Forti EMP, Barbalho-Moulim MC, Rasera-Junior I. Study on pulmonary volumes and thoracoabdominal mobility in morbidly obese women undergoing bariatric surgery, treated with two different physical therapy methods. Rev Bras Fisioter 2009;13(4):294-301.

13. Sprung J, Gajic O, Warner DO. Review article: age related alterations in respiratory function - anesthetic considerations. Can J Anaesth 2006;53(12):1244-57.

14. Clarke MS. The effects of exercise on skeletal muscle in the aged. J Musculoskeletal Neuronal Interact 2004;4(2):175-8.

15. Thompson LV. Age-related muscle dysfunction. Exp Gerontol 2009;44(1-2):106-11.

16. Oyarzún M. Función respiratoria en la senectud. Rev Méd Chile 2009;137(3):411-8.

17. Watsford ML, Murphy AJ, Pine MJ, Coutts AJ. The effect of habitual exercise on respiratorymuscle function in older adults. J Aging Phys Act 2005;13(1):34-44.

18. Freitas FS, Parreira VF, Ibiapina CC. Clinical application of peak cough flow: a literature review. Fisioter Mov 2010;23(3):495-502.
19. Freitas FS, Ibiapina CC, Alvim CG, Britto RR, Parreira VF. Relationship between cough strength and functional level in elderly. Rev Bras Fisioter 2010;14(6):470-6.

20. Summerhill EM, Angov N, Garber C, McCool FD. Respiratory muscle strength in the physically active elderly. Lung 2007;185(6):315-20.

21. Bemben MG. Age-related alterations in muscular endurance. Sports Med 1998;25(4):259-69.

22. Peres PCN, Kojina TY. Uso de eletroestimulação transcutânea diafragmática em pós-operatório de revascularização do miocárdio. Rev Saúde e Pesquisa 2009;2(1):53-7.

23. Forti EMP, Pachani GP, Montebelo MIL, Costa D. Eletroestimulação diafragmática transcutânea em indivíduos saudáveis. Fisioter Bras 2005;6(4):261-4.

24. Neder JA, Andreoni S, Lerario MC, Nery LE. Reference values for lung function tests. II. Maximal respiratory pressures and voluntary ventilation. Braz J Med Biol Res 1999;32(6):719-27.

25. Gonçalves MP, Tomaz CAB, Cassiminho ALF, Dutra MF. Avaliação da força muscular inspiratória e expiratória em idosas praticantes de atividade física e sedentárias. Rev Bras Ciênc Mov 2006;14(1):37-44.

26. Watsford M, Murphy A. The effects of respiratorymuscle training on exercise in older women. J Aging Phys Act 2008;16(3):245-60. 